\title{
Blockade of ICAM-1 Improves the Outcome of Polymicrobial Sepsis via Modulating Neutrophil Migration and Reversing Immunosuppression
}

\author{
Yan-jun Zhao, ${ }^{1,2}$ Wen-jing Yi, ${ }^{1}$ Xiao-jian Wan, ${ }^{1}$ Jun Wang, ${ }^{1}$ Tian-zhu Tao, \\ Jin-bao Li, ${ }^{1}$ Jia-feng Wang, ${ }^{1}$ and Xiao-ming Deng ${ }^{1}$ \\ ${ }^{1}$ Department of Anesthesiology and Intensive Care, Changhai Hospital, Second Military Medical University, 168 Changhai Road, \\ Shanghai 200433, China \\ ${ }^{2}$ Department of Anesthesiology and Intensive Care, Shanghai Ninth People's Hospital, Shanghai Jiaotong University, \\ 639 Zhizaoju Road, Shanghai 200011, China
}

Correspondence should be addressed to Jia-feng Wang; jfwang@smmu.edu.cn and Xiao-ming Deng; deng_x@yahoo.com

Received 10 January 2014; Revised 13 April 2014; Accepted 14 April 2014; Published 6 May 2014

Academic Editor: Martin Hoenigl

Copyright (C) 2014 Yan-jun Zhao et al. This is an open access article distributed under the Creative Commons Attribution License, which permits unrestricted use, distribution, and reproduction in any medium, provided the original work is properly cited.

\begin{abstract}
Intercellular adhesion molecule-1 (ICAM-1) is a key adhesion molecule mediating neutrophil migration and infiltration during sepsis. But its role in the outcome of sepsis remains contradictory. The current study was performed to investigate the role of anti-ICAM-1 antibody in the outcome of polymicrobial sepsis and sepsis-induced immune disturbance. Effect of anti-ICAM-1 antibody on outcome of sepsis induced by cecal ligation and puncture (CLP) was evaluated by the survival analysis, bacterial clearance, and lung injury. Its influence on neutrophil migration and infiltration, as well as lymphocyte status, in thymus and spleen was also investigated. The results demonstrated that ICAM-1 mRNA was upregulated in lung, thymus, and spleen of CLP mice. Anti-ICAM-1 antibody improved survival and bacterial clearance in CLP mice and attenuated lung injury. Migration of neutrophils to peritoneal cavity was enhanced while their infiltration into lung, thymus, and spleen was hampered by ICAM-1 blockade. Anti-ICAM-1 antibody also prevented sepsis-induced apoptosis in thymus and spleen. Positive costimulatory molecules including CD28, CD80, and CD86 were upregulated, while negative costimulatory molecules including PD-1 and PD-L1 were downregulated following anti-ICAM-1 antibody administration. In conclusion, ICAM-1 blockade may improve outcome of sepsis. The rationale may include the modulated neutrophil migration and the reversed immunosuppression.
\end{abstract}

\section{Introduction}

Sepsis refers to the systemic inflammatory response syndrome (SIRS) induced by infection. Severe sepsis, a more serious condition, is the combination of sepsis and dysfunction of at least one organ [1]. Despite the development of medical techniques, mortality of severe sepsis remains high which is over $40 \%$ according to the epidemiological studies from different countries [2-5]. Sepsis also costs a large amount of economic resources all over the world. New therapies are urgent for intervention of the progression of sepsis $[2,3]$.

Disturbance of the immune system is one of the most important features of sepsis, characterized by overwhelming inflammatory responses and dysfunction of the immune cells $[6,7]$. Both anti-inflammatory agents and immuneenhancing treatment show ideal therapeutic effect in animals studies $[8,9]$, but none of these measures has been demonstrated to be effective in clinical trials [10]. The balance between anti- and proinflammatory responses becomes a key point in treating sepsis.

Intracellular adhesion molecule-1 (ICAM-1), also called CD54, is one of the mediators involved in leukocyteendothelial interaction. After neutrophil rolling along the endothelium, CD18 complex on leukocyte may bind to ICAM-1 and promote adhesion and migration of leukocyte toward chemotactic agents [11]. It was reported that inhibition of ICAM-1 expression in lungs was associated with improvement of sepsis induced by cecal ligation and puncture 
(CLP) in mice, when they were treated by some agents such as protein kinase C-delta, hypertonic saline solution, and perfluorocarbon [12-14]. However, the direct role of ICAM-1 in polymicrobial sepsis remained controversial. Several studies used anti-ICAM-1 antibody or gene-deficiency animals to investigate the direct role of ICAM-1 in sepsis, but inconsistent results were found among them [15-18]. Some studies revealed that blockade of ICAM-1 decreased the survival rate in septic animals $[15,16]$, while others showed a beneficial role of ICAM-1 deficiency $[17,18]$. van Griensven et al. [17] argued that the different model might be the reason of the contradictory results because some early studies use a model of bacterial injection, but they used a CLP model. However, Que et al. [15] identified that anti-ICAM-1 antibody or gene deficiency did not improve lung injury in the CLP model either.

Since ICAM-1 is a proadhesion molecule, its blockade using a specific antibody may hamper the proper migration of immune cells and development of lymphocyte. Thus, our present study was performed firstly to confirm the effect of ICAM-1 on polymicrobial sepsis and secondly to detect the apoptotic rate and expression levels of costimulatory molecules in thymus and spleen to clarify the effect of ICAM1 on status of immune cells.

\section{Materials and Methods}

2.1. Mice and Cecal Ligation and Puncture Model. All animal experiments were approved by the Animal Care and Use Committee of Changhai Hospital. Male 8- to 10-week-old C57BL/6 mice (22-30 g) were purchased from the Animals Experimentation Center of Second Military Medical University. All mice were conditioned to the environment under controlled temperature $\left(20 \pm 2^{\circ} \mathrm{C}\right)$, humidity $(60 \pm 5 \%)$, and $12 \mathrm{~h}$ light/12 h dark cycle for one week before surgery.

CLP model was established as described previously [19]. In brief, mice were anesthetized with 2-3\% sevoflurane and a midline abdominal incision was made after disinfecting the abdomen. After exposure, cecum was ligated with a 1-0 Prolene thread and punctured once with a 22-gauge needle. Then the cecum was replaced into the abdomen, and the peritoneal wall was closed in two layers. Sham-operated animals underwent similar laparotomy without ligation and puncture on the cecum. All animals were resuscitated by a subcutaneous injection of $1 \mathrm{~mL}$ sterile physiologic saline solution immediately after the surgery.

2.2. Drug Administration. Mice were randomly divided into four groups; (1) sham group: mice underwent the sham operation and received normal saline $(200 \mu \mathrm{L}$ per mouse); (2) saline group: mice were subjected to CLP and received normal saline (200 $\mu \mathrm{L}$ per mouse); (3) anti-ICAM-1 group: mice were subjected to CLP and intravenously injected with a functional grade purified anti-ICAM-1 antibody (eBioscience, USA) $(50 \mu \mathrm{g} / 200 \mu \mathrm{L}$ per mouse, about $2 \mathrm{mg} / \mathrm{kg}) ;$ (4) isotype group: mice were subjected to CLP and intravenously injected with rat IgG2a isotype antibody (eBioscience, USA)
(50 $\mu \mathrm{g} / 200 \mu \mathrm{L}$ per mouse). Mice receive intravenously antiICAM-1 antibody, isotype antibody, or normal saline immediately after surgery.

2.3. Detection of $m R N A$ by Reverse Transcription. Total RNA was extracted from the lung, thymus, and spleen using the TRIzol Reagent (Invitrogen) and the phenol-chloroform method $24 \mathrm{~h}$ after surgery. The cDNA was synthesized from total RNA in ABI PCR system using a PrimeScript 1st Strand cDNA Synthesis Kit (TaKaRa, China). The murine primer sequences are shown as follows:

ICAM-1 (forward, F) 5'-ACAGACACTAGAGGAGTGAGCAGG-3' and (reverse, R) $5^{\prime}$-GTGAGCGTCCATATTTAGGCATGG-3';

CD28 (F) 5'-CGGGAATGGGAATTTTACCT-3' and (R) $5^{\prime}$-TTGACGTGCAGATTCCAGAG-3';

CD80 (F) $5^{\prime}$-CCATGTCCAAGGCTCATTCT-3' and (R) $5^{\prime}$-TTCCCAGCAATGACAGACAG-3';

CD86 (F) 5'-TCAGTGATCGCCAACTTCAG-3' and (R) $5^{\prime}$-TTAGGTTTCGGGTGACCTTG-3';

PD-1 (F) $5^{\prime}$-GGAGCAGAGCTCGTGGTAAC- $3^{\prime}$ and (R) $5^{\prime}$-TACCAATGACCATGCCTTGA-3';

PD-L1 (F) 5' -TGCTGCATAATCAGCTACGG-3' and (R) $5^{\prime}$-GCTGGTCACATTGAGAAGCA-3';

GAPDH (F) 5'-GGTCCTCAGTGTAGCCCAAG-3' and (R) $5^{\prime}$-AATGTGTCCGTCGTGGATCT-3'.

The real-time PCR were conducted in the StepOnePlus Real-time PCR System (Applied Biosystems, USA) using a SYBR Premix Ex Taq II kit. PCR procedures were referred to the instruction provided by the manufacturer. The levels of mRNA were expressed as fold changes after normalization to GAPDH.

2.4. Survival Analysis and Bacterial Clearance. To evaluate the potential therapeutic effect of anti-ICAM-1 antibody against sepsis, 40 mice were randomly allocated to sham group, saline group, anti-ICAM-1 group, and isotype group; $n=10$ for each group. Survival rates were assessed over the subsequent 7 days.

Blood and peritoneal lavage fluid (PLF) samples were collected $24 \mathrm{~h}$ after surgery from another 24 mice. Blood was collected by heart puncture after isoflurane anesthesia; $n=6$ for each group. PLF was harvested after injection of $2 \mathrm{~mL}$ PBS into the peritoneum. Then $100 \mu \mathrm{L}$ aliquot of each dilution was spread on a tryptic soy agar (TSA) blood agar plate. All plates were incubated at $37^{\circ} \mathrm{C}$ for $24 \mathrm{~h}$. Colonies were counted and the primary concentration of bacteria was quantified by multiplying with the dilution times. The bacterial burden was expressed as colony forming units (CFUs)/mL for all the samples.

2.5. Flow Cytometry. Neutrophil counts in PLF, thymus, and spleen $24 \mathrm{~h}$ after surgery were determined to investigate the migration of neutrophils; $n=6$ for each group. The 
total cell number was counted after lysis of erythrocytes (for spleen and thymus, single-cell suspension was prepared). The neutrophil number was counted by staining with fluorochrome-conjugated anti-Gr-1 antibody. Cells were subjected to fluorescence-activated cell sorting and cell numbers were calculated by flow cytometry (MACSQuant, Miltenyi, German).

2.6. Histopathological Studies. The lungs of the mice in the four groups mentioned above were harvested $24 \mathrm{~h}$ after surgery for histopathological staining. These organ tissues were fixed in buffered formaldehyde (10\% in PBS) for more than $8 \mathrm{~h}$, dehydrated in graded ethanol, and embedded in paraffin. Four-micrometer sections were cut and paraffin was removed by xylene. The tissue sections were then stained with the hematoxylin and eosin reagent and observed under light microscopy. The criteria for scoring lung injury were as follows: 0 , normal tissue; 1 , minimal inflammatory change; 2 , no obvious damage to the lung architecture; 3 , thickening of the alveolar septae; 4 , formation of nodules or areas of pneumonitis that distorted the normal architecture; 5, total obliteration of the field. The slides were examined by two pathologists who were unaware of the groups.

\subsection{Measurement of the Wet-to-Dry Weight Ratio of Lung} Tissues. Twenty-four hours after surgery, one lobe of the right lung was isolated, and the blood and water from the lung surface were removed to determine the wet weight. After the lobe was placed at $80^{\circ} \mathrm{C}$ for 48 hours, the dry weight was determined and the wet-to-dry ratio was calculated.

2.8. Myeloperoxidase (MPO) Activity Assay. The lungs were homogenized, centrifuged $\left(40,000 \mathrm{rpm}, 30 \mathrm{~min}, 4^{\circ} \mathrm{C}\right)$, and resuspended in $50 \mathrm{mM} \mathrm{KPO}_{4}$ buffer (PH 6.0) with $0.5 \%$ hexadecyltrimethylammonium bromide $24 \mathrm{~h}$ after surgery. Then, samples were sonicated and incubated at $60^{\circ} \mathrm{C}$ for $2 \mathrm{~h}$. Later, samples were assayed for activity in a $\mathrm{H}_{2} \mathrm{O}_{2} / \mathrm{O}$ dianisidine buffer at $460 \mathrm{~nm}$ with a spectrophotometer (Shanghai Precision \& Scientific Instrument Co. Ltd, China). Results are expressed as units of MPO activity per gram of lung tissue.

2.9. Quantification of Apoptosis in the Spleen and Thymus. The thymus and spleen were harvested from septic and shamoperated mice and fixed with $10 \%$ buffered formalin $24 \mathrm{~h}$ after surgery. ApopTag Plus Peroxidase In Situ Apoptosis Detection Kit (Chemicon Billerica, MA, USA) was used for terminal deoxynucleotidyl transferase-mediated dUTP nick end labeling (TUNEL) staining. The sections of thymus and spleen were incubated in equilibration buffer for 10 minutes and then terminal deoxynucleotidyl transferase and dUTP-digoxigenin were added to the sections and incubated in a $37^{\circ} \mathrm{C}$ humidified chamber for $1 \mathrm{~h}$. After stopping the reaction, the slices were washed and incubated with antidigoxigenin-peroxidase solution, colorized with $\mathrm{DAB} / \mathrm{H}_{2} \mathrm{O}_{2}$, and counterstained with bisbenzimide. Two investigators examined the samples in a blinded fashion. The percentage of the TUNEL-positive cells was used to represent the apoptosis rate.

2.10. Statistical Analysis. Data are expressed as the mean \pm standard deviation (SD). All statistical analyses were performed in SPSS 16.0. Student's $t$-test was used for comparison between 2 groups and one-way analysis of variance was used for comparison between 3 or more groups. Kaplan-Meier method was utilized to compare the survival rate. A $P$ level less than 0.05 was considered statistically significant.

\section{Result}

3.1. ICAM-1 mRNA Expression Is Upregulated in Lung, Thymus, and Spleen Tissue. CLP-induced polymicrobial sepsis resulted in upregulation of ICAM-1 in different organs $24 \mathrm{~h}$ after CLP surgery. As shown in Figure 1, mRNA levels of ICAM-1 increased more than 2-fold in lung, thymus, and spleen of CLP mice compared with those in sham-operated mice.

3.2. ICAM-1 Blockade Improved Survival and Bacterial Clearance in CLP Mice. The 7-day survival was assessed to investigate whether anti-ICAM-1 antibody protected mice from CLP-induced mortality. Survival of the mice in the anti-ICAM-1 group at the 7th day after surgery was $60 \%$, significantly higher than in the CLP group (30\%) and the isotype group (20\%) (Figure 2(a)). Bacterial clearance was assessed in blood and PLF $24 \mathrm{~h}$ after surgery. Bacterial burden in blood and PLF was almost neglectable in the anti-ICAM1 group, much lower than in the CLP group and the isotype group (Figure 2(b)).

3.3. Anti-ICAM-1 Antibody Treatment Attenuated SepsisInduced Lung Injury. The histologic analysis of lung tissue showed that the lungs obtained from saline treated CLP mice were characterized by neutrophil infiltration, alveolar collapse, consolidation, and occasional alveolar hemorrhage. But anti-ICAM-1 antibody treatment significantly attenuated lung injury with intact pulmonary structure compared with isotype or saline treatment (Figure 3(a)). To further quantify the extent of lung edema, wet-to-dry weight ratio of lungs was measured $24 \mathrm{~h}$ after surgery. Reduced wet-to-dry weight ratio was observed in anti-ICAM-1 antibody treated mice than in isotype antibody and saline treated mice (Figure 3(b)).

3.4. Neutrophil Migration Was Modulated by Anti-ICAM1 Antibody Treatment. Neutrophil migration was regarded as an important factor for killing bacteria, and it was also involved in pathogenesis of organ dysfunction induced by sepsis. Thus, we compared the MPO activity or neutrophil counts to evaluate the migratory ability of neutrophils in different loci $24 \mathrm{~h}$ after surgery. The assay of MPO activity in lung homogenate revealed that MPO activity in antiICAM-1 antibody treated mice was significantly inhibited compared with that in isotype antibody or saline treated mice (Figure 4(a)). Flow cytometry showed that neutrophil counts were elevated in PLF but reduced in thymus and spleen in 

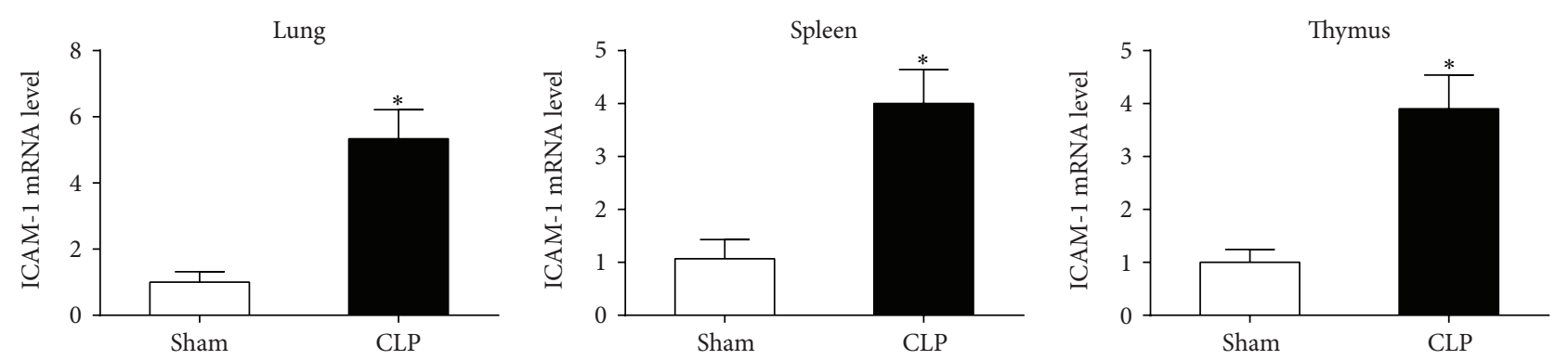

FIgURE 1: The expression levels of ICAM-1 mRNA were upregulated in lung, thymus, and spleen of CLP mice $24 \mathrm{~h}$ after CLP surgery. $n=6$ per each group. ${ }^{*} P<0.01$ when septic mice were compared with sham-operated mice. Bars represent the mean \pm SD.

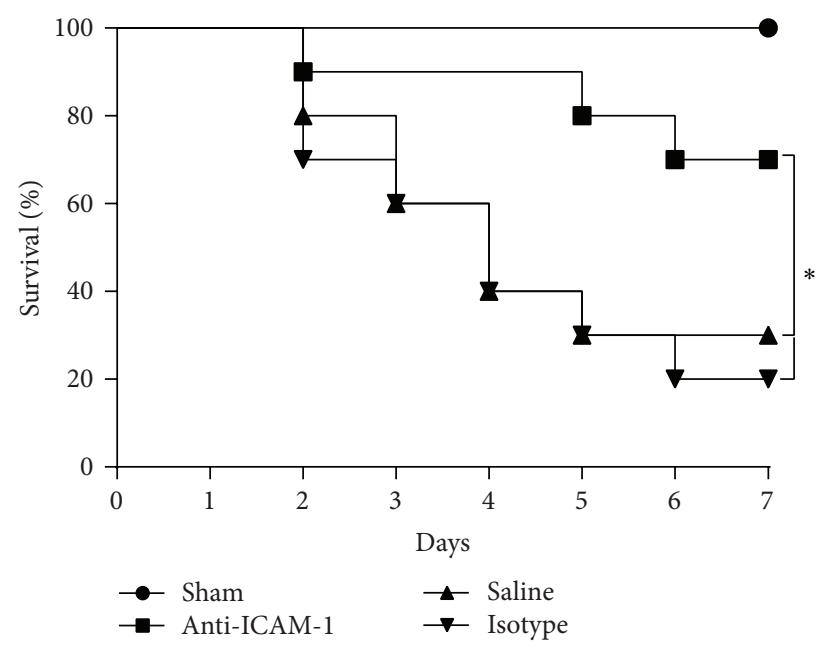

(a)
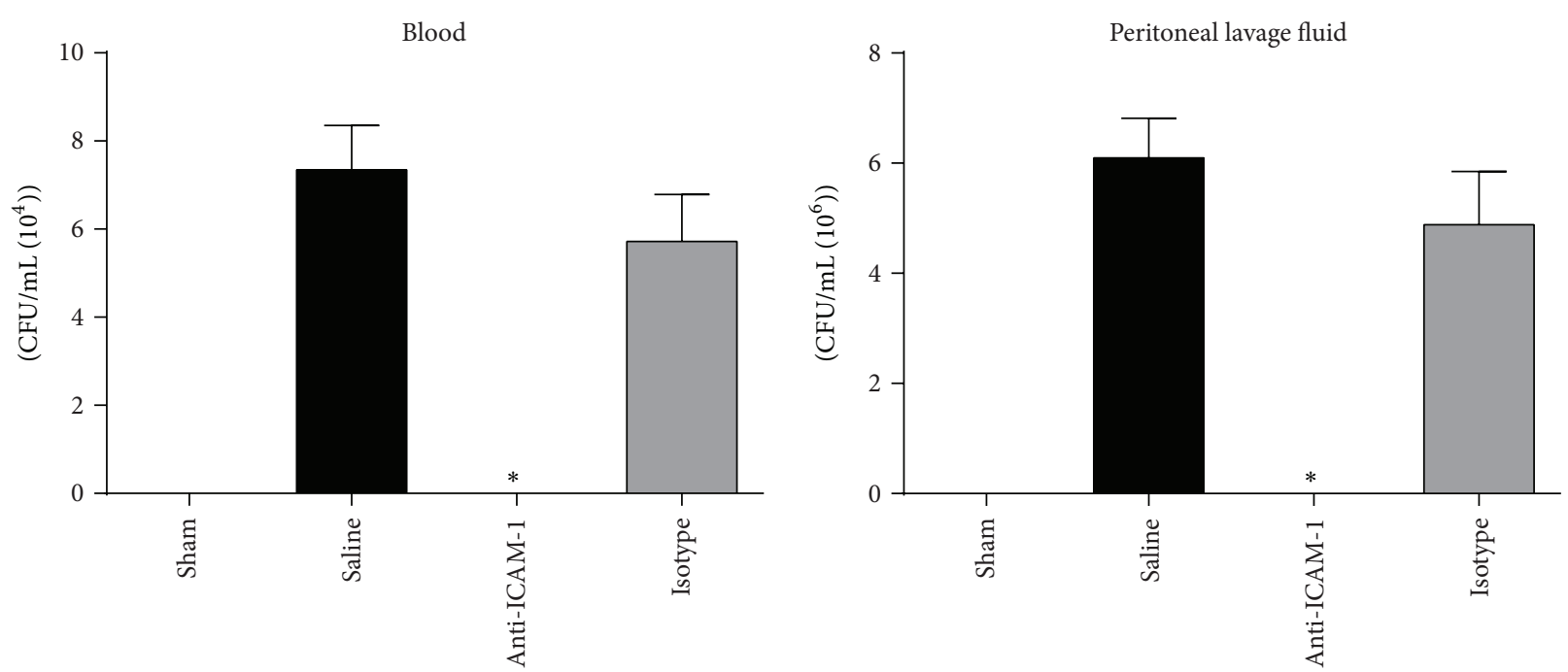

(b)

FIgURE 2: Anti-ICAM-1 antibody treatment improved survival (a) and bacterial clearance (b) in CLP mice. (a) The 7-day survival rates were compared among anti-ICAM-1 antibody treated CLP mice, isotype antibody treated CLP mice, saline treated mice, and sham-operated mice. $n=10$ per each group. ${ }^{*} P<0.05$ when anti-ICAM-1 antibody treated mice were compared with mice treated with isotype antibody or saline. (b) Bacterial clearance was compared among anti-ICAM-1 antibody treated CLP mice, isotype antibody treated CLP mice, saline treated mice, and sham-operated mice $24 \mathrm{~h}$ after surgery. $n=6$ per each group. PLF: peritoneal lavage fluid. ${ }^{*} P<0.05$ when anti-ICAM- 1 antibody treated mice were compared with mice treated with isotype antibody or saline. Bars represent the mean \pm SD. 

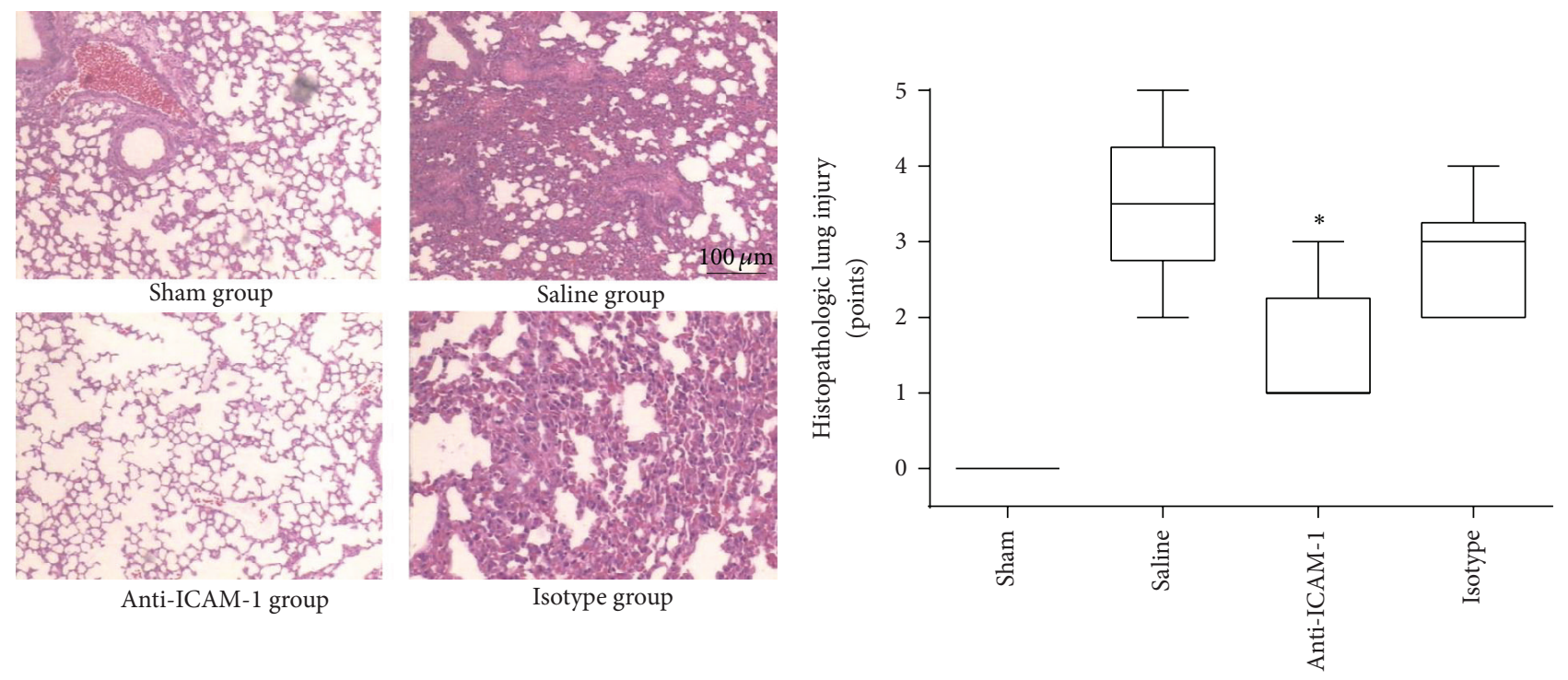

(a)

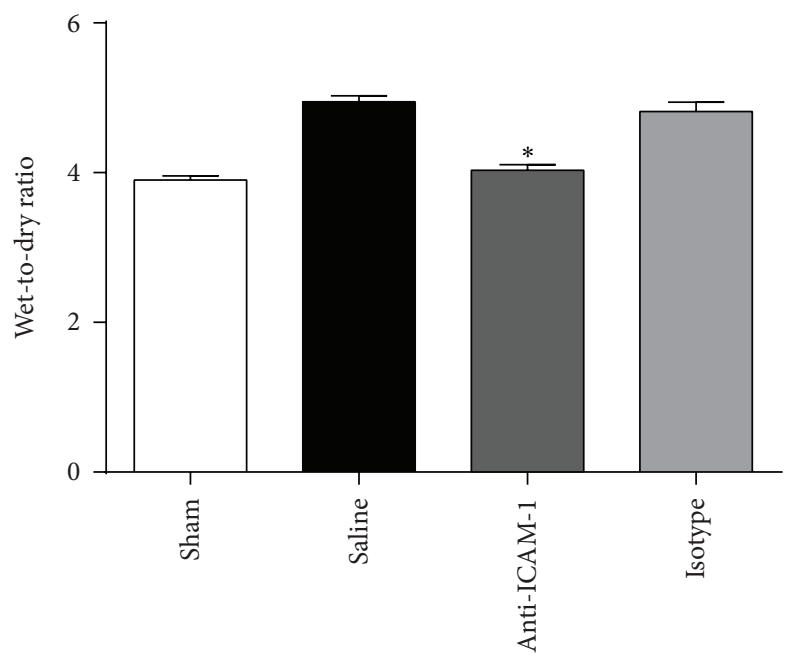

(b)

FIgURE 3: Anti-ICAM-1 antibody attenuated sepsis-induced lung injury as demonstrated by histopathological tests and wet-to-dry weight ratio. (a) The lungs of the anti-ICAM-1 antibody treated CLP mice, isotype antibody treated CLP mice, saline treated CLP mice, and shamoperated mice were harvested $24 \mathrm{~h}$ after surgery for histopathological staining. Histopathological tests showed milder impairment in lungs in anti-ICAM-1 group than in isotype and CLP groups. $n=6$ per each group. (b) Lung wet-to-dry weight ratio was reduced in anti-ICAM-1 group than in isotype and saline groups. ${ }^{*} P<0.05$ when anti-ICAM-1 antibody treated mice were compared with mice treated with isotype antibody or saline. Bars represent the mean \pm SD.

anti-ICAM-1 group compared with that in the isotype and saline groups (Figure 4(b)).

\subsection{Anti-ICAM-1 Antibody Improved the Immune Status} in Thymus and Spleen. In order to investigate the effect of anti-ICAM-1 antibody on immune status, apoptosis and expression levels of costimulatory molecules in spleen and thymus were determined by TUNEL assay and RTPCR, respectively. TUNEL assay showed that apoptotic cells were significantly less in anti-ICAM-1 group than in isotype and saline groups (Figure 5(a)) $(P<0.05)$. Positive costimulatory molecules, including CD28, CD80, and CD86, were all elevated (Figure 5(b)), while negative costimulatory molecules, including PD-1 and PD-L1, were reduced in thymus and spleen of anti-ICAM-1 antibody treated mice according to the RT-PCR assays (Figure 5(c)) $(P<0.05)$.

\section{Discussion}

Our present study reveals that ICAM-1 mRNA is upregulated in lung, thymus, and spleen during sepsis. Blockade of ICAM-1 using a specific antibody improves survival and bacterial clearance and attenuates sepsis-induced lung injury. 


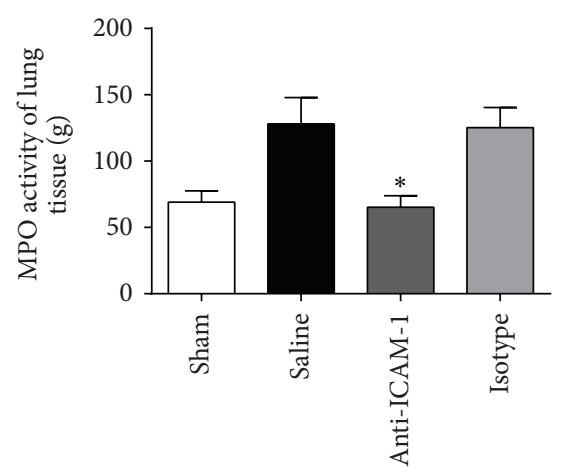

(a)
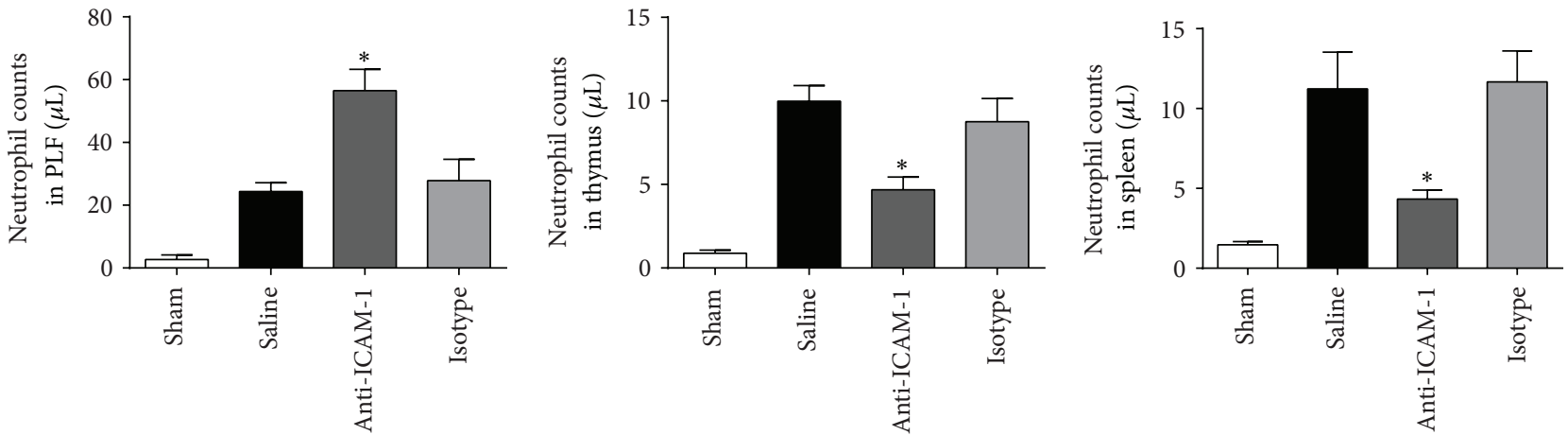

(b)

FIgURE 4: Anti-ICAM-1 blockade modulated neutrophils migration during sepsis. (a) MPO activity in lung homogenate was inhibited in the anti-ICAM-1 group; $n=6$ per each group. ${ }^{*} P<0.05$ when anti-ICAM-1 antibody treated mice were compared with mice treated with isotype antibody or saline. Bars represent the median value and percentiles. (b) Neutrophil counts were elevated in PLF but reduced in thymus and spleen in the anti-ICAM-1 group compared with that in the isotype or saline groups, as demonstrated by flow cytometry. $n=6$ per each group. ${ }^{*} P<0.05$ when anti-ICAM-1 antibody treated mice were compared with mice treated with isotype antibody or saline. Bars represent the mean \pm SD.

Neutrophil infiltration into nonspecific target organs such as lung, thymus, and spleen is modified, while its migration to the peritoneal cavity is enhanced. Less infiltration of neutrophils into thymus and spleen may relieve sepsisinduced lymphocyte apoptosis and disturbance of costimulatory molecules, thereby modulating the immune dysfunction during sepsis.

Neutrophils are the first line of defense against bacterial infection. They respond to pathogens rapidly and produce reactive oxygen species to degrade bacteria [20]. Patients with neutropenia are believed to be more vulnerable to infection [21]. Unfortunately, activation and mobilization of neutrophils are always systemic during sepsis. At the same time of killing pathogens, neutrophils cause tissue damage as well. In the local site of infection, damage of tissue may be manifested as abscess. But in the remote nonspecific organs, organ dysfunction may develop, such as sepsis-induced lung injury, kidney injury, hepatic injury, and myocardial injury [22]. Removal of neutrophils from patients with systemic inflammation by filters has been shown to improve respiratory and renal function [23].

ICAM-1 is one of the most important adhesion molecules determining the adhesion and migration of neutrophils to target organs. However, overexpression of ICAM-1 on endothelial cells from nonspecific organs may be a leading cause of sepsis-induced organ dysfunction. Although we did not determine the protein level of ICAM-1, upregulation of ICAM-1 in organs had been well defined in many studies [24]. According to the study of Basit et al., anti-ICAM-1 reduced neutrophil recruitment in alveolar space after lipopolysaccharide (LPS) challenge by more than $50 \%$. Antagonism of its ligand, CD11b, also reverses the endothelial injury caused by sepsis [25]. In our present study, anti-ICAM-1 antibody did attenuate sepsis-induced lung injury, as demonstrated by the histopathological test and wet-to-dry weight ratio.

However, it is very strange that early and late studies showed the absolutely converse effects of ICAM-1 on the outcome of sepsis. Two studies performed near the year of 2000 excluded any beneficial effect of anti-ICAM-1 antibody or ICAM-1 gene deficiency $[15,16]$. There was even evidence showing a harmful effect on septic lung injury [15]. But two studies published in 2005 [18] and 2006 [17] demonstrated a protective role of ICAM-1 deficiency in polymicrobial sepsis. There might be some difference in techniques that we did not understand, including antibody craft and genedeficiency technology. Anyway, there seemed to be more lines of evidence proving the protective role of ICAM-1 blockade in outcome of sepsis. 

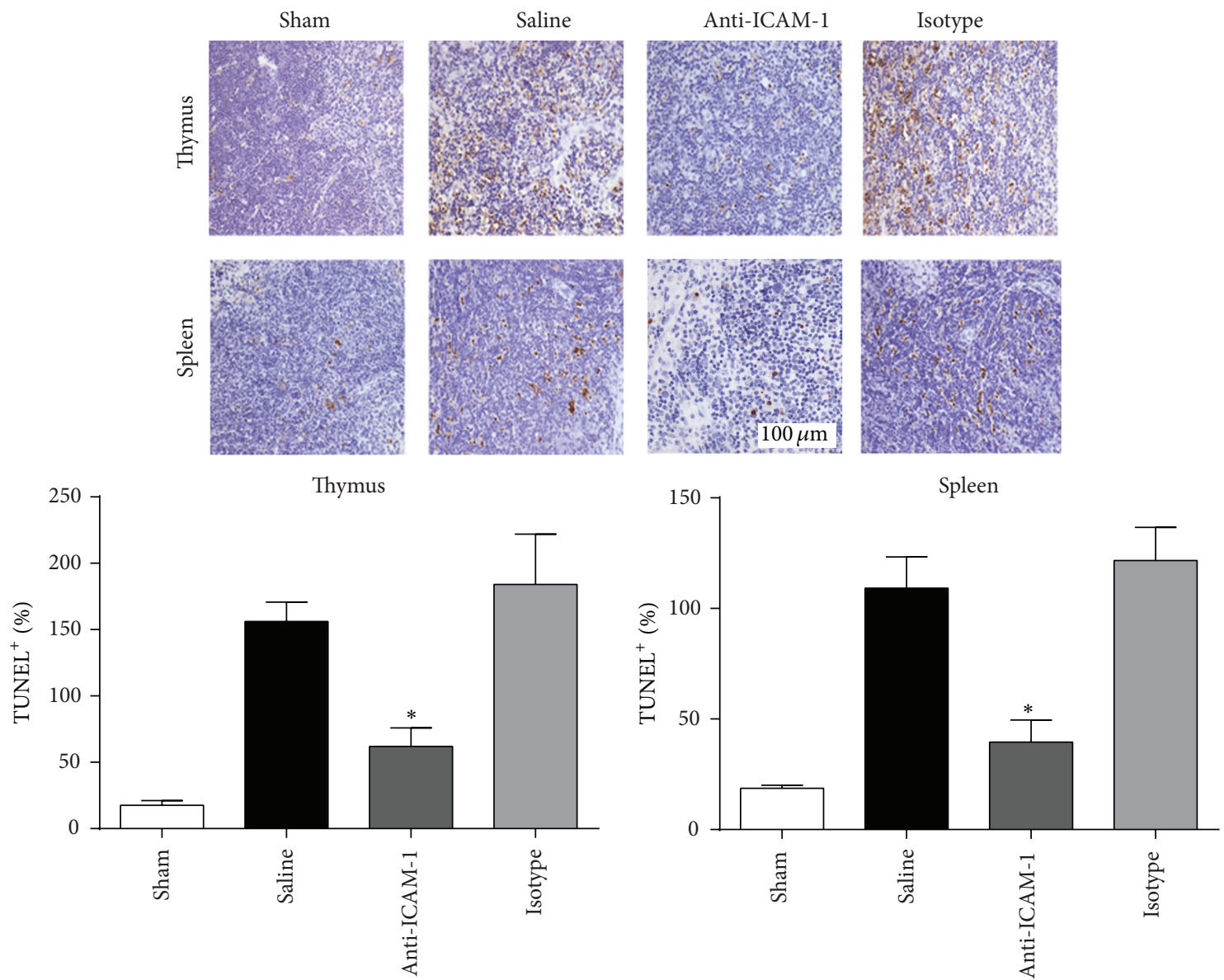

(a)
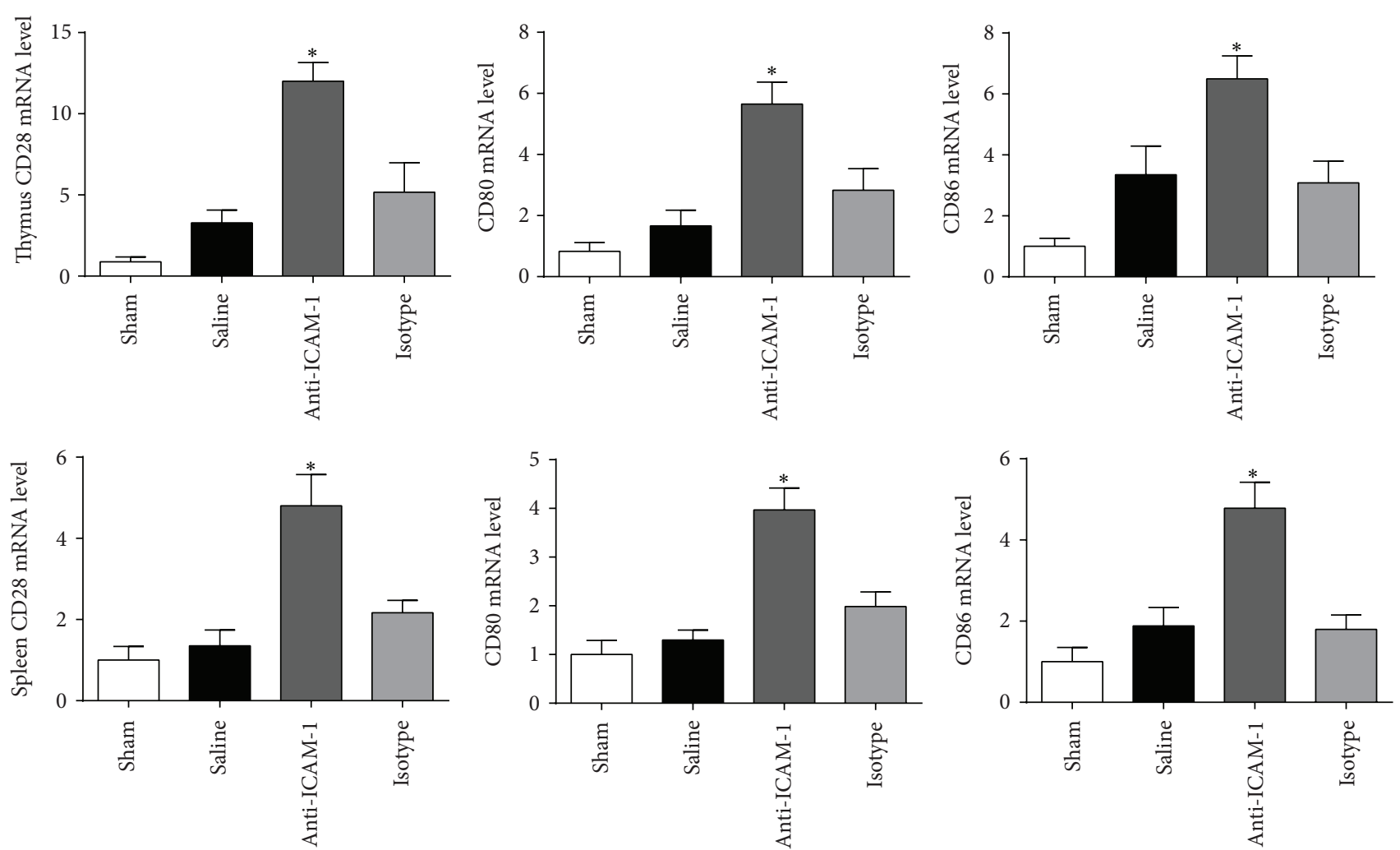

(b)

Figure 5: Continued. 

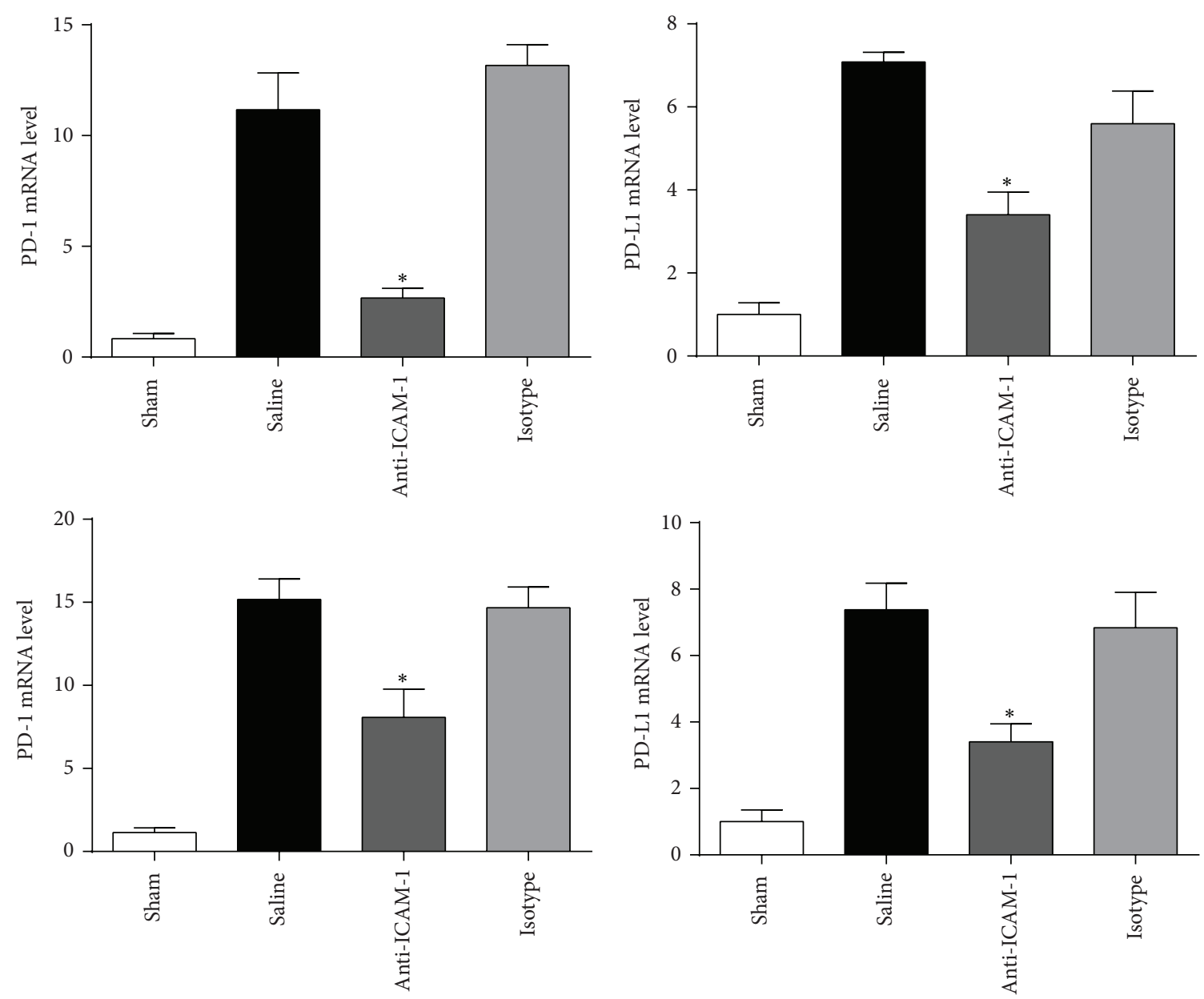

(c)

FIGURE 5: Effect of ICAM-1 blockade on the immune status in thymus and spleen. (a) Representative sections analyzed by the in situ TUNEL assays. Apoptotic cells were significantly reduced by anti-ICAM-1 treatment in thymus and spleen. (b) RT-PCR assays of costimulatory molecules in thymus and spleen, including CD28, CD80, CD86, PD-1, and PD-L1. $n=6$ per each group. ${ }^{*} P<0.05$ when anti-ICAM-1 antibody treated mice were compared with mice treated with isotype antibody or saline. Bars represent the mean \pm SD.

Modulation of neutrophil migration is a highlight in this study. Neutrophil paralysis is involved in the inability of bacterial clearance and organ dysfunction [26]. The modulatory effect of anti-ICAM-1 antibody enhances the migratory capacity of neutrophils to the infection locus while attenuating its infiltration to nonspecific organs. Chemotaxis of neutrophils is mediated by several factors, such as CXC chemokine receptors and C-C motif receptors. Unfortunately, most of the chemokine receptors are not specific and contribute to the impaired organs [27]. But our data showed that reduced neutrophil adhesion to endothelial cells by anti-ICAM-1 antibody increased the specificity of neutrophil migration. The chemokines for the chemokine receptors included several agents, such as ATP and cytokines. We speculated that inhibited neutrophil infiltration into nonspecific organs might reduce the concentration of chemokines in these organs but the concentration in infection locus remained unchanged. Therefore, migratory capacity of neutrophils towards infectious site was relatively enhanced by
anti-ICAM-1 antibody. But we only evaluated the histological change of lung and liver, while dysfunction of other organs was also important during the pathogenesis of sepsis, such as heart, liver, and kidney. The role of anti-ICAM-1 antibody in sepsis-induced dysfunction of other organs remained to be further investigated.

It was also interesting to notice that lymphocyte apoptosis and disturbance of costimulatory molecules were reversed by anti-ICAM-1 antibody. Meanwhile, infiltration of neutrophils to the thymus and spleen was also attenuated, which might be the reason of the protective role of anti-ICAM-1 antibody in immune system. Immune dysfunction has been considered a main cause of death in septic patients and animals recently, manifested as lymphocyte apoptosis and disturbance of costimulatory molecules. Upregulation of coinhibitory molecules, PD-L1 and PD-1, and downregulation of positive costimulatory molecules, CD28, CD80, and CD86, are the features of sepsis-induced immunosuppression, reversal of which will improve the survival of septic mice $[28,29]$. 
Recent studies tended to discover an inhibitory effect of neutrophils on lymphocyte. Pillay et al. [30] identified a new subset of neutrophils in human systemic inflammation, which inhibited lymphocyte proliferation stimulated by phytohemagglutinin. The marker of these neutrophils is $\mathrm{CD} 11 \mathrm{c}^{\text {bright }} / \mathrm{CD} 62 \mathrm{~L}^{\mathrm{dim}} / \mathrm{CD} 11 \mathrm{~b}^{\text {bright }} / \mathrm{CD} 16^{\text {bright }}$ and the inhibitory effect was mediated by the integrin Mac-1 (CD11b/CD18 complex). Neutrophils might also inhibit lymphocyte via other signals, as reported by the same group investigating the Mac-1, such as the coinhibitory signaling of PD-L1/PD-1 pathway. Interferon- $\gamma$ upregulates the PDL1 expression level on neutrophils, by which lymphocyte proliferation is inhibited [31]. Therefore, less infiltration of neutrophils into thymus and spleen might improve the immune environment of immune organs and reverse sepsisinduced lymphocyte dysfunction by attenuating neutrophillymphocyte interactions.

\section{Conclusion}

In conclusion, our present study demonstrates that elevation of ICAM-1 in lung, thymus, and spleen is detrimental in sepsis. Blockade of ICAM-1 using a neutralizing antibody attenuates sepsis-induced death and lung injury. Anti-ICAM1 antibody modulates the migration and infiltration of neutrophils, which further contributes to less organ impairment and preserved lymphocyte function.

\section{Conflict of Interests}

The authors declare that they have no conflict of interests to disclose.

\section{Authors' Contribution}

Yan-jun Zhao, Wen-jing Yi, and Xiao-jian Wan contributed equally to this work.

\section{Acknowledgments}

This work was supported by the National Natural Science Foundation of China (Grant no. 81201493), Natural Science Foundation of Shanghai (12ZR1454000), and Key Project on Basic Research of Shanghai (12JC1410700).

\section{References}

[1] M. M. Levy, M. P. Fink, J. C. Marshall et al., “2001 SCCM/ESICM/ACCP/ATS/SIS International Sepsis Definitions Conference," Critical Care Medicine, vol. 31, no. 4, pp. 1250-1256, 2003.

[2] G. S. Martin, D. M. Mannino, S. Eaton, and M. Moss, "The epidemiology of sepsis in the United States from 1979 through 2000," New England Journal of Medicine, vol. 348, no. 16, pp. 1546-1554, 2003.

[3] B. Cheng, G. Xie, S. Yao et al., "Epidemiology of severe sepsis in critically ill surgical patients in ten university hospitals in China," Critical Care Medicine, vol. 35, no. 11, pp. 2538-2546, 2007.
[4] C. Engel, F. M. Brunkhorst, H.-G. Bone et al., "Epidemiology of sepsis in Germany: results from a national prospective multicenter study," Intensive Care Medicine, vol. 33, no. 4, pp. 606-618, 2007.

[5] K. E. Sands, D. W. Bates, P. N. Lanken et al., "Epidemiology of sepsis syndrome in 8 academic medical centers," Journal of the American Medical Association, vol. 278, no. 3, pp. 234-240, 1997.

[6] R. S. Hotchkiss, C. M. Coopersmith, J. E. McDunn, and T. A. Ferguson, "The sepsis seesaw: tilting toward immunosuppression," Nature Medicine, vol. 15, no. 5, pp. 496-497, 2009.

[7] L. P. Skrupky, P. W. Kerby, and R. S. Hotchkiss, "Advances in the management of sepsis and the understanding of key immunologic defects," Anesthesiology, vol. 115, no. 6, pp. 13491362, 2011.

[8] R. Bhargava, C. J. Altmann, A. Andres-Hernando et al., "Acute lung injury and acute kidney injury are established by four hours in experimental sepsis and are improved with pre, but not post, sepsis administration of TNF- $\alpha$ antibodies," PLoS ONE, vol. 8, Article ID e79037, 2013.

[9] J. C. Hebert and M. O’Reilly, “Granulocyte-macrophage colonystimulating factor (GM-CSF) enhances pulmonary defenses against pneumococcal infections after splenectomy," Journal of Trauma. Injury, Infection and Critical Care, vol. 41, no. 4, pp. 663-666, 1996.

[10] R. K. Root, R. F. Lodato, W. Patrick et al., "Multicenter, double-blind, placebo-controlled study of the use of filgrastim in patients hospitalized with pneumonia and severe sepsis," Critical Care Medicine, vol. 31, no. 2, pp. 367-373, 2003.

[11] J. G. Moreland, R. M. Fuhrman, J. A. Pruessner, and D. A. Schwartz, "CD11b and intercellular adhesion molecule1 are involved in pulmonary neutrophil recruitment in lipopolysaccharide-induced airway disease," American Journal of Respiratory Cell and Molecular Biology, vol. 27, no. 4, pp. 474480, 2002.

[12] M. J. Mondrinos, T. Zhang, S. Sun et al., "Pulmonary endothelial protein kinase C-delta (PKC $\delta$ ) regulates neutrophil migration in acute lung inflammation," American Journal of Pathology, vol. 184, pp. 200-213, 2014.

[13] M. C. Theobaldo, F. Llimona, R. C. Petroni, E. C. Rios, I. T. Velasco, and F. G. Soriano, "Hypertonic saline solution drives neutrophil from bystander organ to infectious site in polymicrobial sepsis: a cecal ligation and puncture model," PLoS ONE, vol. 8, Article ID e74369, 2013.

[14] S. Hou, H. Ding, Q. Lv et al., "Therapeutic effect of intravenous infusion of perfluorocarbon emulsion on LPS-induced acute lung injury in rats," PLoS ONE, vol. 9, Article ID e87826, 2014.

[15] L. G. Que, B.-H. Kang, Y.-C. T. Huang, C. A. Piantadosi, and L.-Y. Chang, "Anti-intercellular adhesion molecule-1 antibody and intercellular adhesion molecule-1 gene deficiency do not prevent pulmonary neutrophil recruitment in polymicrobial sepsis," Shock, vol. 9, no. 4, pp. 304-309, 1998.

[16] K. E. Welty-Wolf, M. S. Carraway, Y.-C. T. Huang et al., "Antibody to intercellular adhesion molecule 1 (CD54) decreases survival and not lung injury in baboons with sepsis," American Journal of Respiratory and Critical Care Medicine, vol. 163, no. 3, pp. 665-673, 2001.

[17] M. van Griensven, C. Probst, K. Müller, P. Hoevel, and H.C. Pape, "Leukocyte-endothelial interactions via ICAM-1 are detrimental in polymicrobial sepsis," Shock, vol. 25, no. 3, pp. 254-259, 2006.

[18] F. Hildebrand, H.-C. Pape, P. Harwood et al., "Role of adhesion molecule ICAM in the pathogenesis of polymicrobial sepsis," 
Experimental and Toxicologic Pathology, vol. 56, no. 4-5, pp. 281290, 2005.

[19] J. Zhu, J. Wang, Y. Sheng et al., "Baicalin improves survival in a murine model of polymicrobial sepsis via suppressing inflammatory response and lymphocyte apoptosis," PLoS ONE, vol. 7, Article ID e35523, 2012.

[20] M. V. Parsey, R. M. Tuder, and E. Abraham, "Neutrophils are major contributors to intraparenchymal lung IL-1 $\beta$ expression after hemorrhage and endotoxemia," Journal of Immunology, vol. 160, no. 2, pp. 1007-1013, 1998.

[21] M. Legrand, A. Max, V. Peigne et al., "Survival in neutropenic Patients with severe sepsis or septic shock," Critical Care Medicine, vol. 40, no. 1, pp. 43-49, 2012.

[22] K. Brown, S. Brain, J. Pearson, J. Edgeworth, S. Lewis, and D. Treacher, "Neutrophils in development of multiple organ failure in sepsis," The Lancet, vol. 368, no. 9530, pp. 157-169, 2006.

[23] D. F. Treacher, M. Sabbato, K. A. Brown, and V. Gant, "The effects of leucodepletion in patients who develop the systemic inflammatory response syndrome following cardiopulmonary bypass," Perfusion, vol. 16, pp. S67-S73, 2001.

[24] K. Ley, "Molecular mechanisms of leukocyte recruitment in the inflammatory process," Cardiovascular Research, vol. 32, no. 4, pp. 733-742, 1996.

[25] A. Basit, J. Reutershan, M. A. Morris, M. Solga, C. E. Rose Jr., and K. Ley, "ICAM-1 and LFA-1 play critical roles in LPS-induced neutrophil recruitment into the alveolar space," American Journal of Physiology. Lung Cellular and Molecular Physiology, vol. 291, no. 2, pp. L200-L207, 2006.

[26] J. C. Alves-Filho, F. Spiller, and F. Q. Cunha, "Neutrophil paralysis in sepsis," Shock, vol. 34, no. 1, pp. 15-21, 2010.

[27] F. O. Souto, J. C. Alves-Filho, W. M. Turato, M. AuxiliadoraMartins, A. Basile-Filho, and F. Q. Cunha, "Essential role of CCR2 in neutrophil tissue infiltration and multiple organ dysfunction in sepsis," American Journal of Respiratory and Critical Care Medicine, vol. 183, no. 2, pp. 234-242, 2011.

[28] Y. Zhang, Y. Zhou, J. Lou et al., "PD-L1 blockade improves survival in experimental sepsis by inhibiting lymphocyte apoptosis and reversing monocyte dysfunction," Critical Care, vol. 14, no. 6, article R220, 2010.

[29] Y. Zhang, J. Li, J. Lou et al., "Upregulation of programmed death-1 on T cells and programmed death ligand-1 on monocytes in septic shock patients," Critical Care, vol. 15, no. 1, article R70, 2011.

[30] J. Pillay, V. M. Kamp, E. van Hoffen et al., "A subset of neutrophils in human systemic inflammation inhibits $\mathrm{T}$ cell responses through Mac-1," Journal of Clinical Investigation, vol. 122, no. 1, pp. 327-336, 2012.

[31] S. de Kleijn, J. D. Langereis, J. Leentjens et al., "IFN- $\gamma$-stimulated neutrophils suppress lymphocyte proliferation through expression of PD-L1," PLoS ONE, vol. 8, Article ID e72249, 2013. 


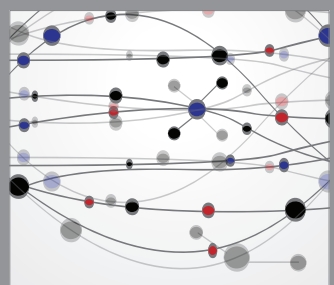

The Scientific World Journal
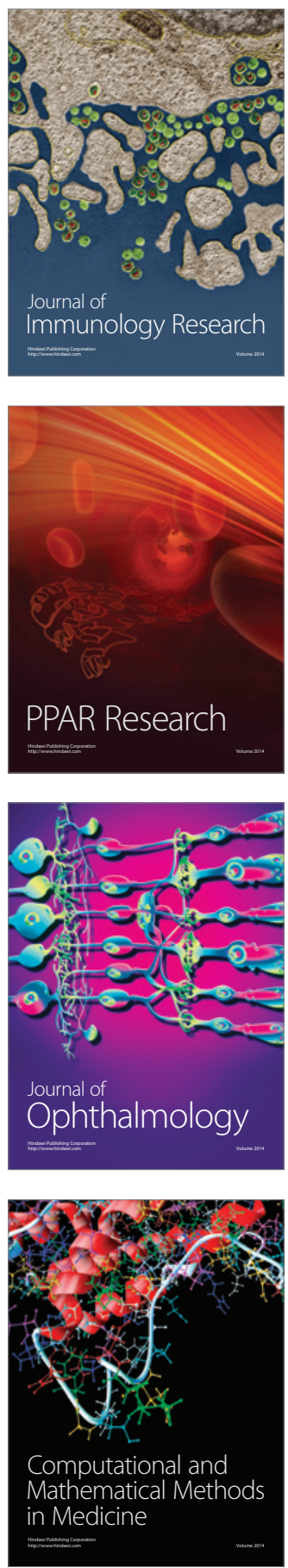

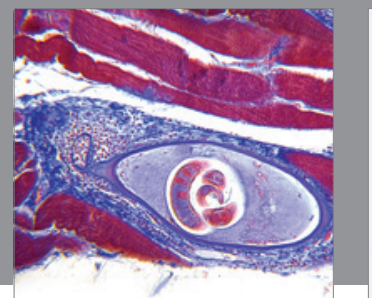

Gastroenterology

Research and Practice
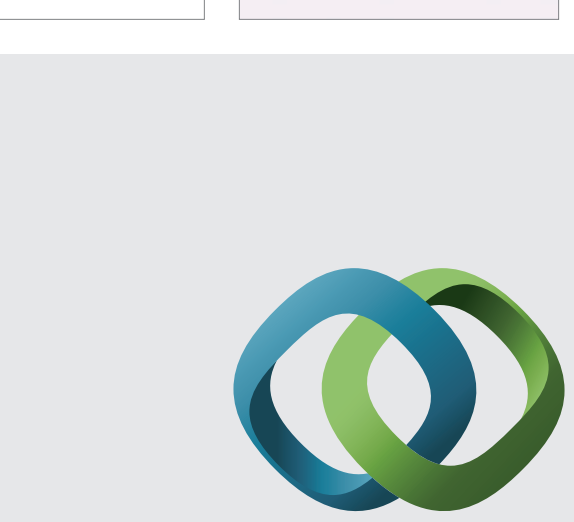

\section{Hindawi}

Submit your manuscripts at

http://www.hindawi.com
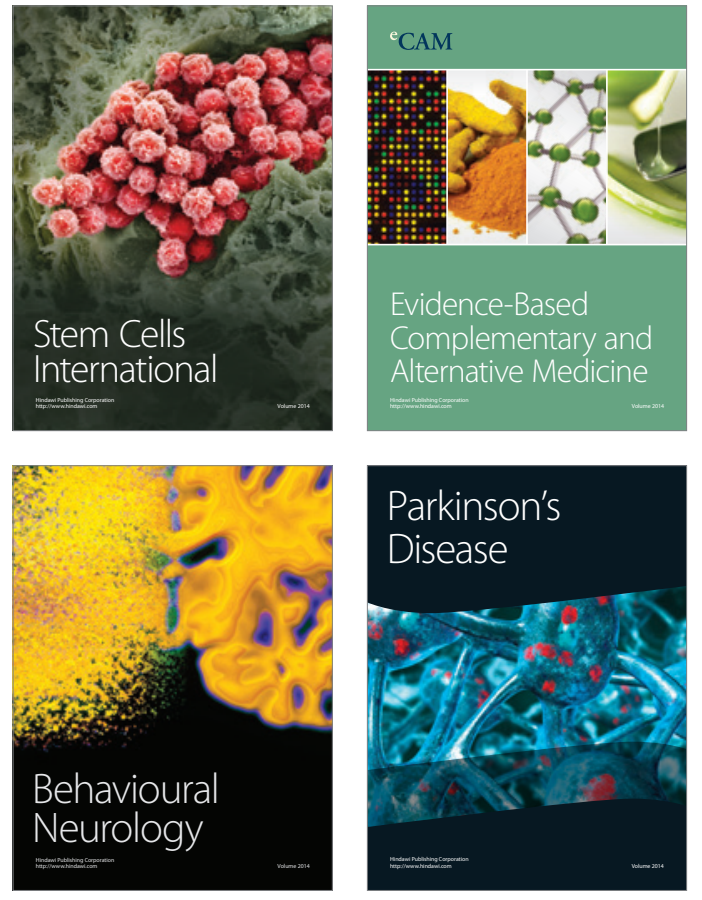
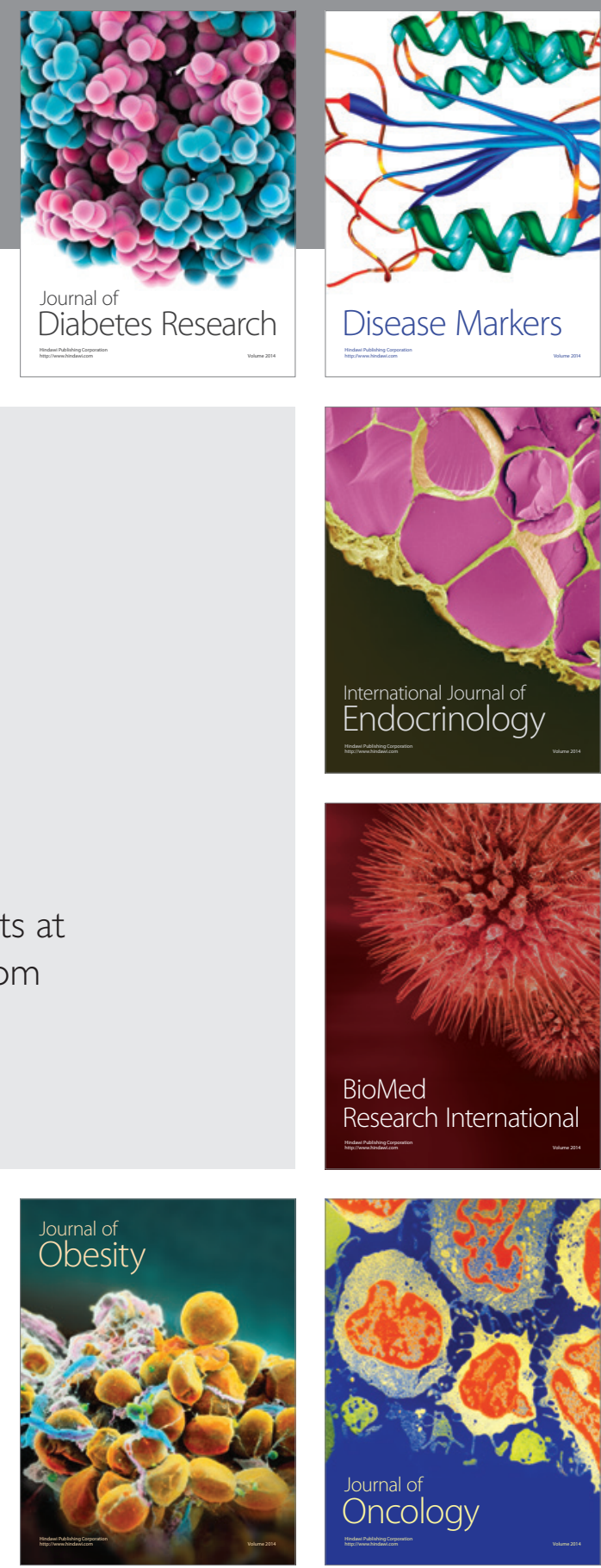

Disease Markers
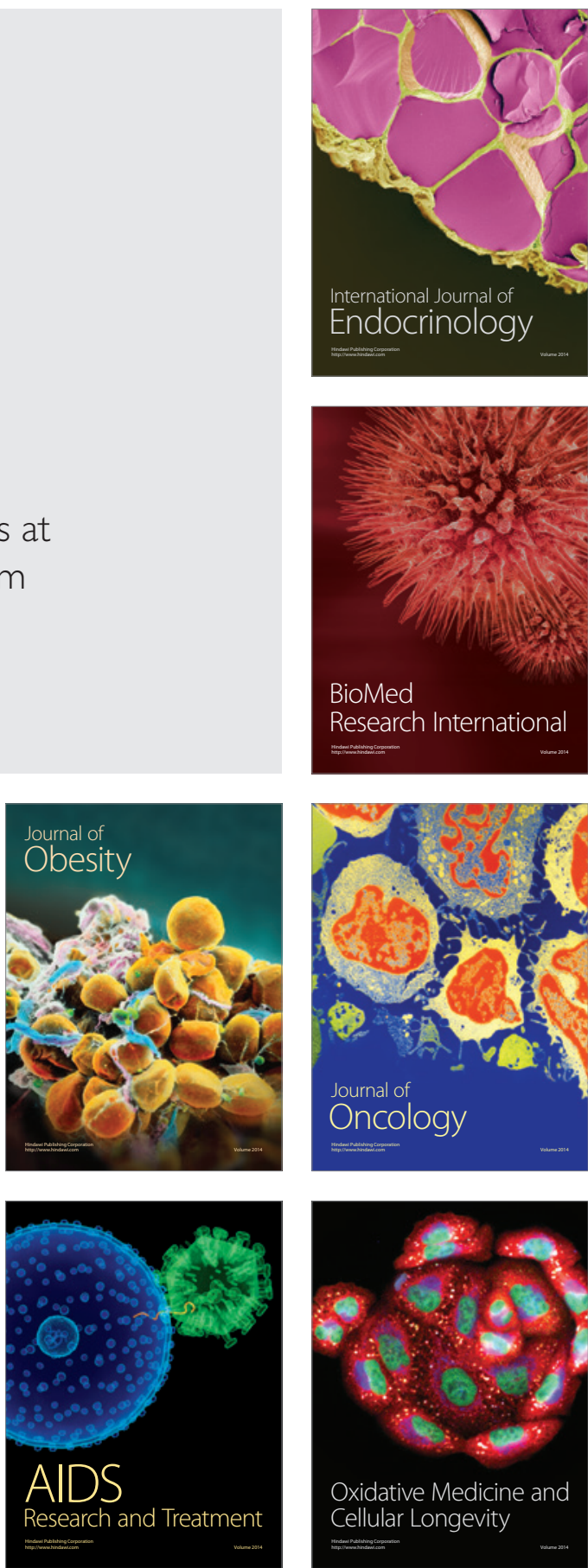